\title{
Healthy Monozygotic Twins Born from a Vitrified Blastocyst Derived from a Vitrified Oocyte, and a Highly Efficient Vitrification for Freezing Human Oocytes and Blastsocysts
}

\author{
Shaohua Huang, Christina Miao, Samuel Sun, Sameh Toma \\ In Vitro Fertilization Lab, North Carolina Center for Reproductive Medicine, Cary, USA \\ Email address: \\ Hualian99@yahoo.com (Shaohua Huang),Ch_miao@yahoo.com (C. Miao), Summysun2000@yahoo.com (S. Sun), \\ drstoma@gmail.com (S. Toma)
}

\section{To cite this article:}

Shaohua Huang, Christina Miao, Samuel Sun, Sameh Toma. Healthy Monozygotic Twins Born from a Vitrified Blastocyst Derived from a Vitrified Oocyte, and a Highly Efficient Vitrification for Freezing Human Oocytes and Blastsocysts. American Journal of BioScience. Vol. 8, No. 5, 2020, pp. 132-138. doi: 10.11648/j.ajbio.20200805.12

Received: November 11, 2019; Accepted: January 15, 2020; Published: September 24, 2020

\begin{abstract}
We used simplified oocyte/embryo vitrification and warming protocols (Irvine Scientific) combined with vitristraws (SciTech Invention) to freeze and thaw human oocytes and blastsocysts. Throughout the year of 2014, twelve recipients were transferred embryos developed from vitrified donor oocytes, and fourteen recipients were transferred embryos developed from fresh donor oocytes at the North Carolina center for reproductive medicine (NCCRM). There were no statistically significant differences in donor age $(25.9 \pm 3.6$ vs $24.9 \pm 3.2)$ and recipient age $(43.0 \pm 5.4$ vs $41.4 \pm 6.8)$, fertilization rates $(86.2 \%$ vs $87.0 \%)$, blastocyst development rates $(50.0 \%$ vs $53.8 \%)$, number of embryo transferred $(1.7 \pm 0.8$ vs $1.9 \pm 0.4)$, clinical pregnancy rates per transfer $(75.0 \%$ vs $71.4 \%)$ and live birth rates per transfer $(66.7 \%$ vs $57.1 \%)$ between vitrified and fresh oocyte cycles, respectively. The results demonstrate that vitrification techniques can be used to cryopreserve human oocytes for future use. We are also reporting the live birth of healthy monozygotic twins resulted from a re-vitrified blastocyst derived from a vitrified oocyte. Oocytes from a 30-year-old donor were vitrified in vitristraws. Seven out of eight oocytes survived after thawing on November 16, 2013. Those seven oocytes were inseminated by intracytoplasmic sperm injection (ICSI) at about 2 hours post thawing. All seven oocytes were tested as fertilized by pronuclear check at 18 hours after ICSI. Those fertilized oocytes showed normal cleavage on day 2 and day 3. Four of them developed to blastsocysts by culturing in continuous single culture medium in a tri-gas incubator for 5 days. Two blastsocysts were transferred to a 43 -yearold recipient, but that did not result in a pregnancy. The other two blastsocysts were re-vitrified in a vitristraw. The re-vitrified blastsocysts were thawed and then transferred to the same recipient on May 8, 2014. The patient achieved a normal pregnancy on her second transfer. On June 14, 2014, an ultrasound scan detected two heartbeats in one gestational sac. Two healthy monozygotic boys (weighing 2466g and 2353g) were born on January 13, 2015. To our knowledge, this is the first report of monozygotic twins born from an embryo by twice vitrification at oocyte and blastocyst stage.
\end{abstract}

Keywords: Human Oocyte, Blastocyst, Vitristraw, Twice Vitrification, Monozygotic Twins

\section{Introduction}

In 1986, the first successful attempt of human oocyte cryopreservation was reported by using slow-freezing [1]. In 1999, a live birth was achieved by vitrification of human oocytes [2]. As vitrification is superior to the slow-freezing [3-5], vitrification techniques have been widely used in cryopreservation of oocytes [5-12] and blastsocysts [13-17] in human in vitro fertilization (IVF). By using DNA fingerprinting, Forman et al have shown that oocyte vitrification does not increase the rate of aneuploidy or diminish the implantation potential of viable blastsocysts [18]. The evidence that aneuploidy rate does not increase in vitrified oocytes is a welcome endorsement of the safety of the vitrification technique and a watershed in assisted reproductive technology [19]. Many studies show that vitrified oocytes result in similar fertilization, development and pregnancy rates compared to fresh oocytes [20-23]. 
Oocyte vitrification techniques can be employed to enable women to use their own eggs in their future pregnancy for medical or social reasons [24-28], and also establish egg banks for donation [29-35]. Two re-vitrified day 2 embryos derived from in-vitro matured and vitrified oocytes were transferred, but there was no pregnancy [35]. Healthy babies born from re-vitrified embryos in donation cycles conducted with vitrified oocytes have been reported $[16,36]$. Moreover, after studying a large series of transfers of vitrified embryos generated from previously vitrified oocytes, Cobo et al show that double vitrification has no impact on delivery rates [37]. We report the first live birth of healthy monozygotic twins (born on January 13, 2015) resulted from a re-vitrified day 5 embryo derived from a vitrified oocyte.

\section{Materials and Methods}

\subsection{Oocyte Retrievals, Fertilization and Embryo Culture}

Oocytes were retrieved from donors through transvaginal ultrasound-guided puncture of the follicles in 36 hours after lupron trigger. Cumulus-oocytes complexes were cultured in insemination medium (IM, human tube fluid (HTF, Irvine Scientific) containing $10 \%$ of serum substitute supplement (SSS, Irvine Scientific)) for about 4 hours in fresh cycles or 2 hours in vitrification cycles. All mature (fresh/vitrified) oocytes were fertilized by ICSI using standard ICSI protocols. Fertilization checks were completed by performing pronuclear checks under a stereo microscope at 18 hours post ICSI. Fertilized oocytes were moved to $45 \mu \mathrm{l}$ droplets (covered with mineral oil) of continuous single culture medium (CSCM, Irvine Scientific) containing $10 \%$ of $\mathrm{SSS}$ in a $60-\mathrm{mm}$ culture dish (Fisher Scientific) in order to culture further for blastocyst development till 5 or 6 days. All cultures were performed at $37^{\circ} \mathrm{C}$ in benchtop incubators (Cook Medical) in the tri-gas of $4 \% \mathrm{O}_{2}, 6 \% \mathrm{CO}_{2}$, and $90 \% \mathrm{~N}_{2}$.

\subsection{Oocyte Cryopreservation}

Two hours after retrieval, cumulus cells were removed from cumulus-oocytes complexes before vitrification, and only mature oocytes in metaphase II (MII) were used for vitrification. Oocytes were vitrified by following the simplified MII oocyte vitrification protocols (Irvine Scientific) and using the Vit Kit-Freeze media (Irvine Scientific) containing washing solution (WS, a HEPES buffered solution of M-199 containing gentamicin sulfate (35 $\mu \mathrm{g} / \mathrm{ml}$ ), and $20 \%$ (v/v) of dextran serum supplement (DSS)), equilibration solution (ES, a HEPES buffered solution of M199 containing gentamicin sulfate $(35 \mu \mathrm{g} / \mathrm{ml}), 7.5 \%(\mathrm{v} / \mathrm{v})$ of each DMSO and ethylene glycol and $20 \%(\mathrm{v} / \mathrm{v})$ of DSS)), and vitrification solution (VS, a HEPES buffered solution of M-199 containing gentamicin sulfate $(35 \mu \mathrm{g} / \mathrm{ml}), 15 \%(\mathrm{v} / \mathrm{v})$ of each DMSO and ethylene glycol and $20 \%(\mathrm{v} / \mathrm{v})$ of DSS and $0.5 \mathrm{M}$ sucrose). All vitrification procedures were done at room temperature $\left(22-27^{\circ} \mathrm{C}\right)$. Oocytes were placed into a 20 $\mu 1$ drop of WS for 1 minute. A $20 \mu 1$ drop of ES was merged to the WS drop, with spontaneous mixing for 2 minutes (using tip of transfer pipette to move ES towards WS until drops merge). The second $20 \mu \mathrm{l}$ ES drop was merged into the WS+ES drop, with spontaneous mixing for other 2 minutes. Then, oocytes were transferred from the merged drop to the third $20 \mu \mathrm{l}$ drop of ES and exposed undisturbed for 6-10 minutes. During the 6-10 minute exposure, a $50 \mu 1$ drop of VS was aseptically dispensed separately. Oocytes were transferred from the third ES drop to the VS drop for 50 seconds before loading, and gently pipetted once within the VS drop to ensure complete rinse in VS. A vitristraw (SciTech Invention) was pre-labeled with the patient's information. Two or three oocytes were loaded to the tip of one vitristraw with about $0.5 \mu \mathrm{l}$ of VS within 10 seconds and then the vitristraw was plunged into the liquid nitrogen (LN2) immediately. The tip of the vitristraw was inserted into a clear sleeve and twisted tightly within LN2.

\subsection{Embryo Cryopreservation}

Embryos were vitrified by following the simplified Embryo vitrification protocols (Irvine Scientific) and using the Vit Kit-Freeze media containing WS, ES and VS. All vitrification procedures were to be done at room temperature $\left(22-27^{\circ} \mathrm{C}\right)$. Embryos were placed into a $50 \mu \mathrm{l}$ drop of WS for 1 minute and then transferred into a $50 \mu 1$ drop of ES for 6-10 minutes. During the 6-10 minute exposure, a $50 \mu$ drop of VS was aseptically dispensed. Embryos were transferred from the ES drop to the VS drop for 50 seconds before loading, and gently pipetted once within VS drop to ensure complete rinse in VS. A vitristraw was pre-labeled with patient's information. One or two embryos were loaded onto the tip of one vitristraw with about $0.5 \mu \mathrm{l}$ of VS within 10 seconds and then the vitristraw was plunged into LN2 immediately. The tip of the vitristraw was inserted into a clear sleeve and twisted tightly within LN2.

\subsection{Oocyte/Embryo Thawed}

Embryos or oocytes were thawed by following the simplified embryo and oocyte warming protocols (Irvine Scientific) and using Vit Kit-Thaw media (Irvine Scientific) containing WS, dilution solution (DS, a HEPES buffered solution of M-199 containing gentamicin sulfate $(35 \mu \mathrm{g} / \mathrm{ml})$, $0.5 \mathrm{M}$ sucrose and $20 \%(\mathrm{v} / \mathrm{v})$ of DSS), and thawing solution (TS, a HEPES buffered solution of M-199 containing gentamicin sulfate $(35 \mu \mathrm{g} / \mathrm{ml}), 1.0 \mathrm{M}$ sucrose and $20 \%(\mathrm{v} / \mathrm{v})$ of DSS). One $\mathrm{ml}$ of TS was aseptically dispensed in the center of an organ dish (Fisher Scientific) and warmed to $37^{\circ} \mathrm{C}$ (in an incubator without $\mathrm{CO}_{2}$ or on a heating stage) at least 30 minutes prior starting warming procedure. The tip of the vitristraw (carrying embryos/oocytes) was separated from the clear sleeve within LN2 and then immediately plunged into TS $\left(37^{\circ} \mathrm{C}\right)$ in the center of the organ dish and gently swirled to detach embryos/oocytes. The embryos/oocytes were then kept in TS for 1 minute at room temperature. Embryos/oocytes were transferred from TS to DS for 4 minutes at room temperature, undisturbed. During the 4 minutes exposure in DS, 2 drops $(50 \mu \mathrm{L})$ of WS (WS1, WS2) 
were aseptically dispensed. Embryos/oocytes were transferred from the DS drop to the WS1 drop for 4 minutes at room temperature, undisturbed, and then moved from the WS1 drop to the WS2 drop for other 4 minutes at room temperature, undisturbed. Embryos were transferred to preequilibrated IM with $20 \%(\mathrm{v} / \mathrm{v})$ of SSS and then cultured at $37^{\circ} \mathrm{C}$ for about 2-4 hours prior to embryo transfer. Oocytes were transferred to pre-equilibrated IM with $20 \%(\mathrm{v} / \mathrm{v})$ of SSS and then cultured at $37^{\circ} \mathrm{C}$ for about 2 hours before ICSI.

\subsection{Embryo Transfer}

Assisted hatching ( $\mathrm{AH}$, creating a hole in the zona pellucid) was performed on all vitrified embryos (or fresh embryos generated from vitrified oocytes) prior to embryo transfer (ET). One or two embryos were transferred to a recipient using a Wallace embryo transfer catheter (Origio Inc.) by ultrasound-guide.

\section{Results}

\subsection{Fresh and Frozen Oocyte Cycles}

From January to December of 2014, twelve vitrified oocyte cycles and fourteen fresh oocyte cycles were performed in our clinic through oocyte donation programs.
After thawing, all oocytes were cultured in IM containing $20 \%$ of SSS for about 2 hours, and only survived oocytes (figure 1) would be inseminated by ICSI (figure 2). Figure 3 shows day 5 blastsocysts developed from fresh oocytes, and figure 4 shows day 5 blastsocysts developed from vitrified oocytes. Morphology of blastsocysts from vitrified oocytes (figure 4) looks as good as that (figure 3) from fresh oocytes. The data were analyzed with a chi-squared test of independence, and P-values lower than or equal to 0.05 were regarded as an indicator of significant difference. The results demonstrate in table 1 that there were no statistically significant differences in donor age $(25.9 \pm 3.6$ vs $24.9 \pm 3.2)$ and recipient age $(43.0 \pm 5.4$ vs $41.4 \pm 6.8)$, embryo number for transfer $(1.7 \pm 1.3$ vs $1.9 \pm 0.9)$ between vitrified and fresh oocyte cycles. The fertilization and blastocyst development rates in the fresh oocyte cycles were higher than that in the vitrified oocyte cycles, $(87.0 \%$ vs $86.2 \%$ and $53.8 \%$ vs $50.0 \%$ respectively); nonetheless, there were no statistically significant differences between two groups. The clinical pregnancy and live birth rates per transfer in vitrified oocyte cycles were higher than that in fresh oocyte cycles $(75.0 \%$ vs $71.4 \%$ and $66.7 \%$ vs $57.1 \%$ respectively), however, there were still no statistically significant differences between two groups.

Table 1. Oocyte donation program in NCCRM in 2014.

\begin{tabular}{llll}
\hline & Vitrified oocyte cycles (N=12) & Fresh oocyte cycles (N=14) & P-value \\
\hline Donor age & $25.9 \pm 3.6$ & $24.8 \pm 3.2$ & 0.406 \\
Recipient age & $43.0 \pm 5.4$ & $41.4 \pm 6.8$ & 0.540 \\
Oocyte survival (\%) (post thawing) & $65 / 90(72.2 \%)$ & N/A & $160 / 184(87.0 \%)$ \\
Fertilization (\%) & $56 / 65(86.2 \%)$ & $86 / 160(53.8 \%)$ \\
Blastocyst development (\%) & $28 / 56(50.0 \%)$ & $1.9 \pm 0.4$ & 0.934 \\
Embryo number for transfer & $1.7 \pm 0.8$ & $10 / 14(71.4 \%)$ \\
Clinical pregnancy (\%) & $9 / 12(75.0 \%)$ & $8 / 14(57.1 \%)$ \\
Live birth (\%) & $8 / 12(66.7 \%)$ & 0.230 \\
\hline
\end{tabular}

\subsection{Monozygotic Twins (a Case Report)}

Oocytes (from a 30-year-old donor) were vitrified in vitristraws in Vit Kit-Freeze media. Seven out of eight oocytes survived after thawing in Vit Kit-Thaw media on November 16,2013 . Those seven oocytes were inseminated by ICSI at about 2 hours post thawing. All seven oocytes were tested as fertilized by pronuclear check at 18 hours after ICSI. All seven fertilized oocytes were cultured in CSCM in a tri-gas incubator and cleaved normally to embryos (figure 5) at day 2, but only four of them developed to blastsocysts (figure 6, figure 7) at day 5. The $\mathrm{AH}$ was performed on two blastsocysts (figure 6) before they were transferred to a 43-year-old recipient, but this did not lead to a pregnancy. The other two blastsocysts (figure 7) were re-vitrified in a vitristraw in Vit Kit-Freeze media. The re-vitrified blastsocysts were thawed in Vit Kit-Thaw media, and $\mathrm{AH}$ was performed. The thawed blastsocysts (figure 8) were then transferred to the same recipient on May 8, 2014. The patient achieved a normal pregnancy on her second transfer. On June 14, 2014, an ultrasound scan detected two heartbeats in one gestational sac. Two healthy monozygotic boys (weighing $2466 \mathrm{~g}$ and $2353 \mathrm{~g}$ ) were born on January 13 , 2015. From a survey in 2018, the two boys were normal growth within 3 years.

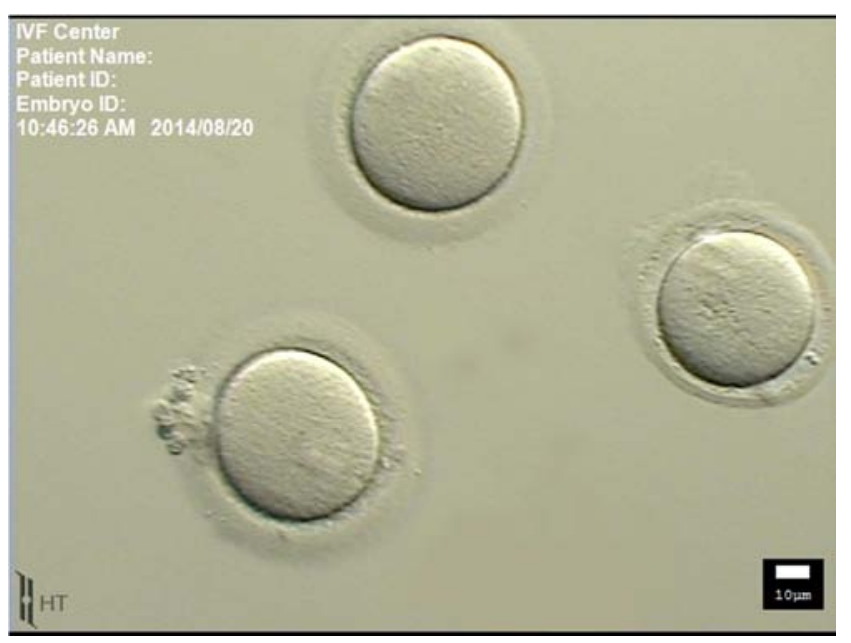

Figure 1. Survived oocytes after vitrification/thawing. 


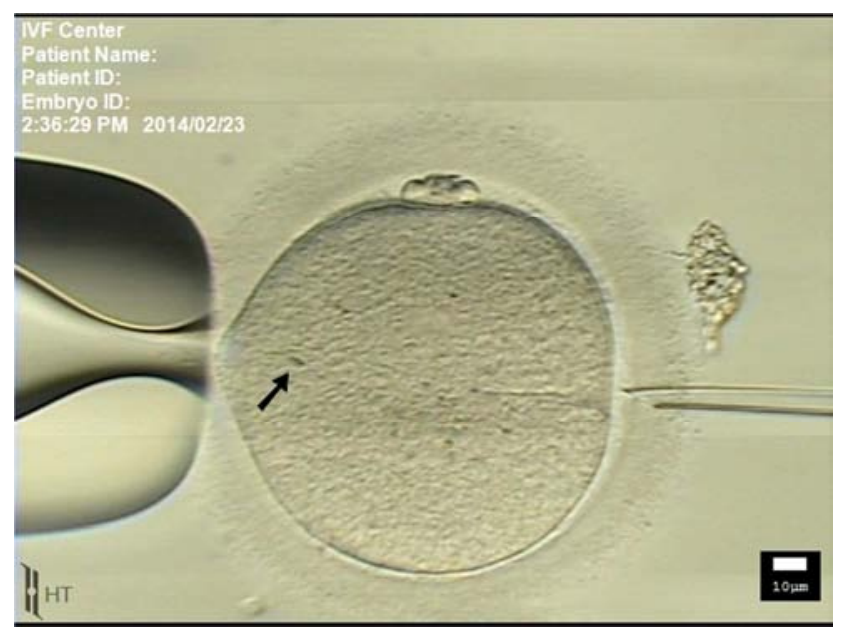

Figure 2. A sperm (arrow) was injected into a vitrified/thawed oocyte by ICSI.

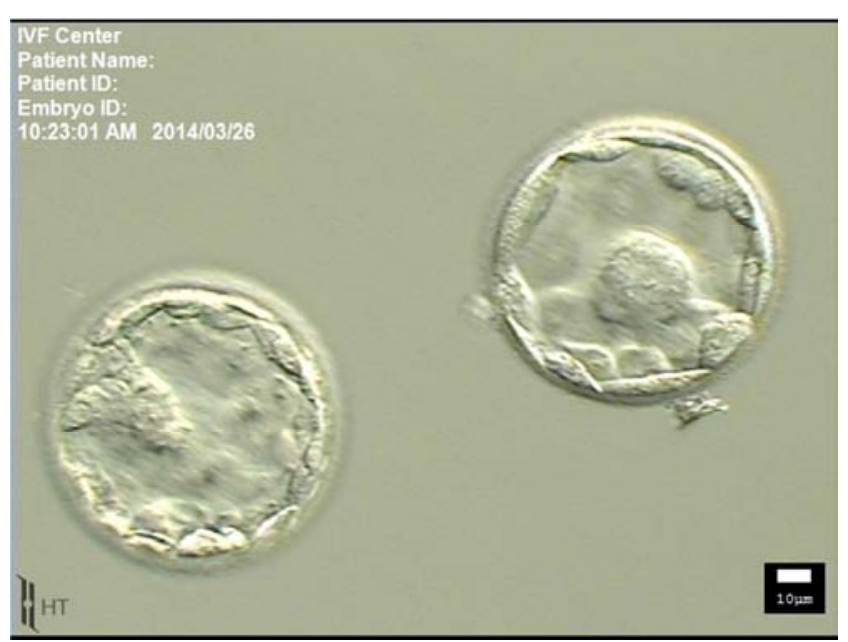

Figure 3. Two day 5 blastsocysts from fresh oocytes.

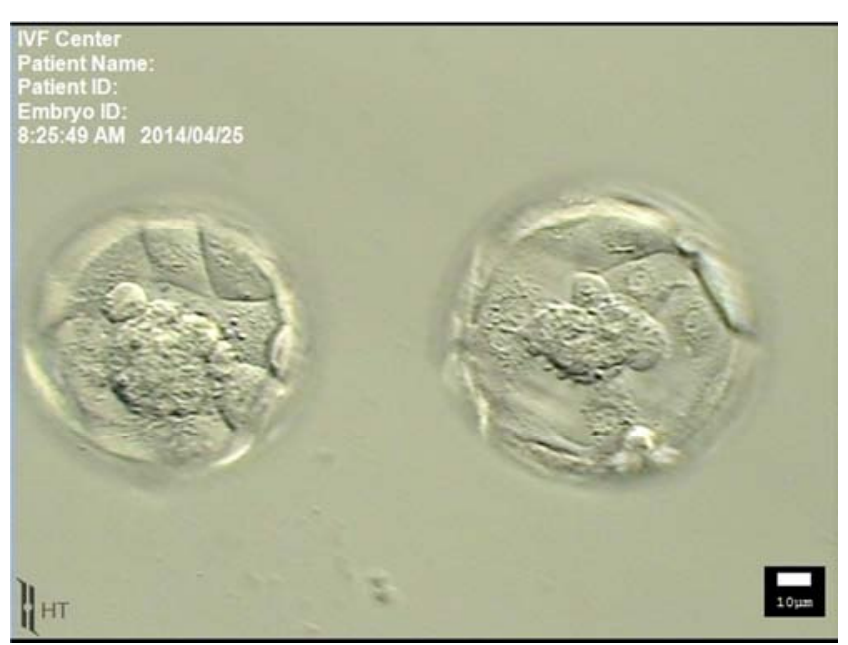

Figure 4. Two day 5 blastsocysts from vitrified oocytes.

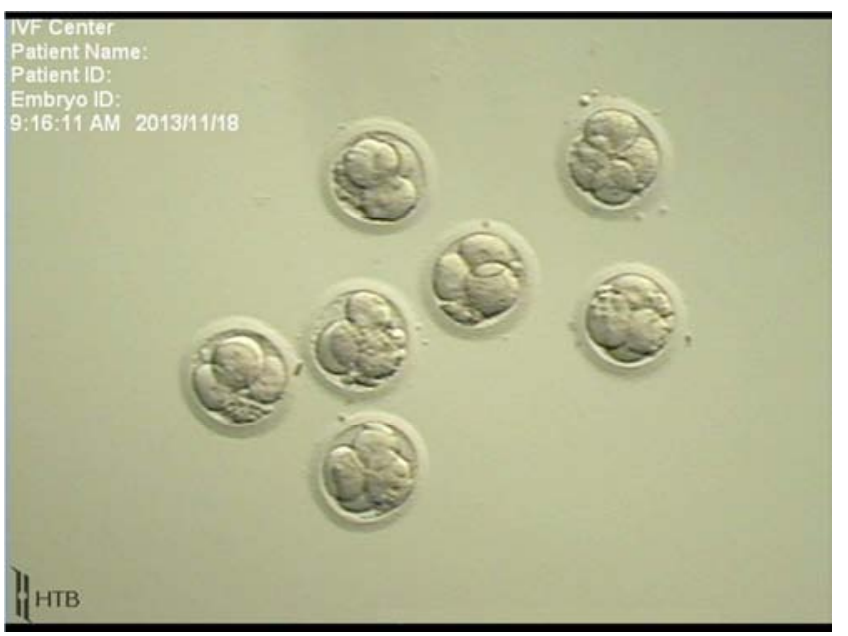

Figure 5. Seven day 2 embryos from vitrified oocytes.

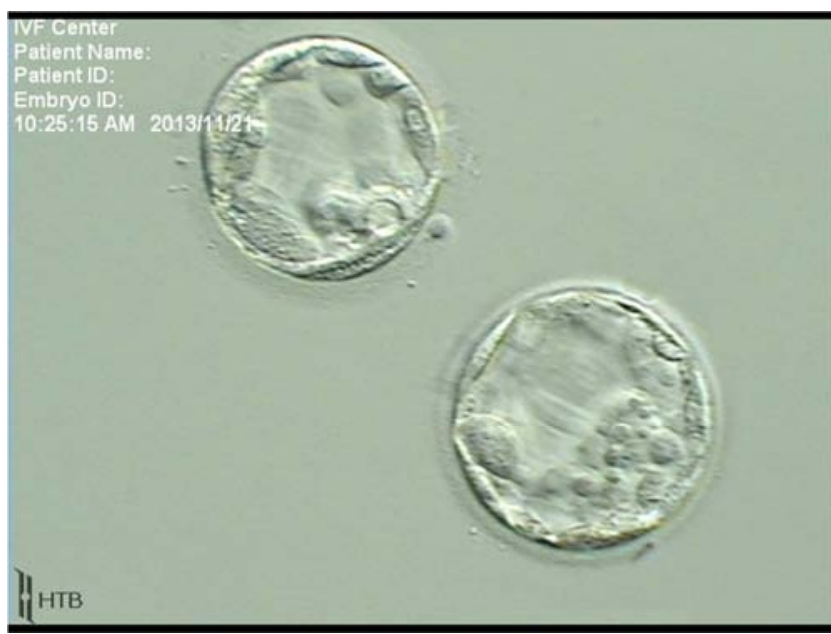

Figure 6. Two day 5 blastsocysts from vitrified oocytes.

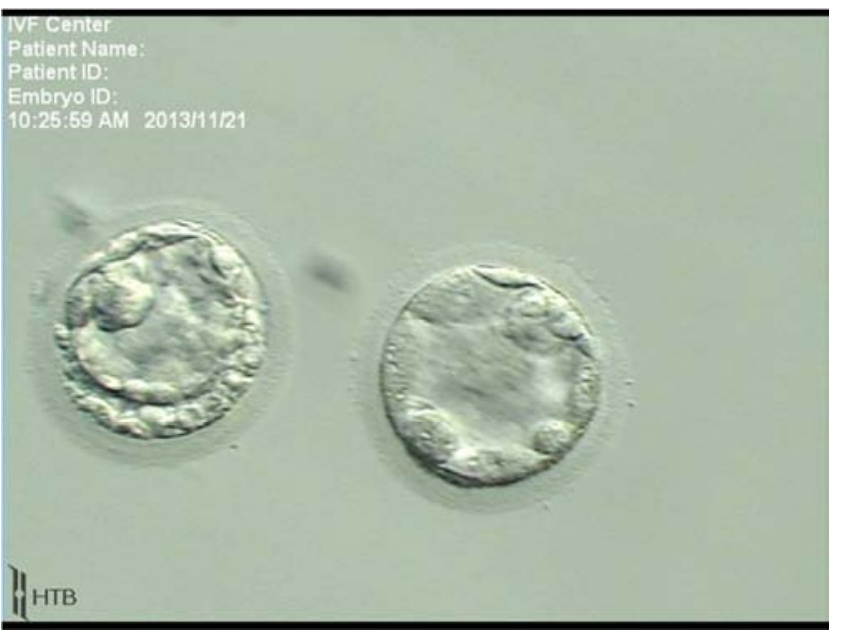

Figure 7. Two day 5 blastsocysts (going to be re-vitrified) from vitrified oocytes. 


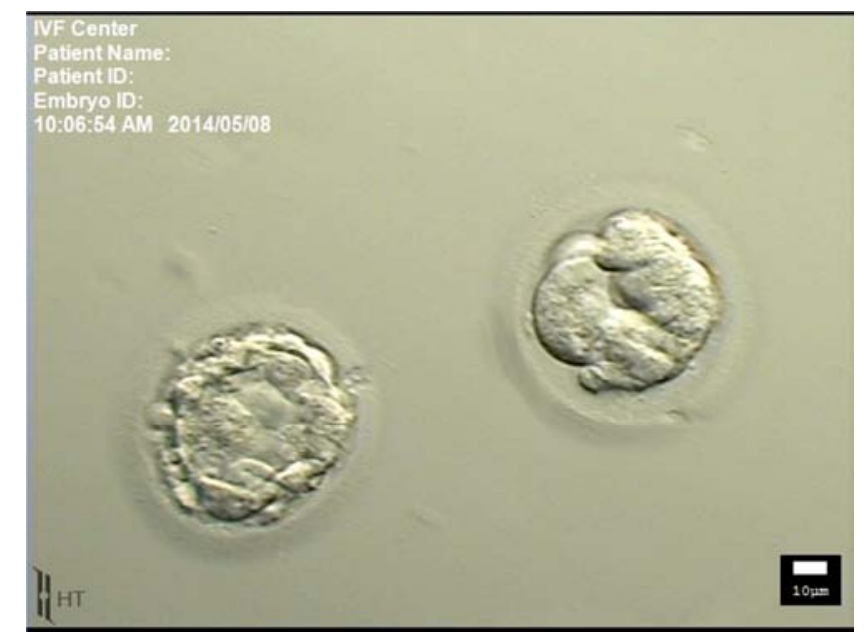

Figure 8. Two thawed blastsocysts after twice vitrification at oocyte and blastocyst stage.

\section{Discussion}

Many devices such as cryoloop [38], cryotip [39], cryotop [19], cryolock [40], cryoleaf [41], HSV [42], Rapid-i [44] and iVirti [41] etc., have been employed as oocyte/embryo carries for vitrification. In this study, we used vitristraws (SciTech Invention) as oocyte/embryo carries for vitrification and achieved a good result of $66.7 \%$ in live birth rate (table 1). Some studies show that vitrified oocytes result in similar fertilization, development and pregnancy rates compared to fresh oocytes [20-23]. Cobo's study demonstrates there was no difference in fertilization $(76.3 \%$ and $82.2 \%)$, day 2 cleavage $(94.2 \%$ and $97.8 \%)$, day 3 cleavage $(80.8 \%$ and $80.5 \%)$, and blastocyst formation $(48.7 \%$ and $47.5 \%)$ for vitrified and fresh oocytes, respectively [20]. From comparing frozen-thawed and fresh donor oocytes in recipients, it has been reported that $92.5 \%$ of vitrified oocytes survived after warming, and there were no statistically significant differences in fertilization rates $(74.2 \%$ vs $73.3 \%)$, clinical pregnancy rates $(55.4 \%$ vs $55.6 \%)$ between vitrified and fresh donor oocyte cycles [29]. Although our vitrified oocyte survival rate $(72.2 \%)$ (table 1 ) was lower than the that (92.5\%) from the study by Cobo et al [29], the fertilization rate $(86.2 \%)$ and clinical pregnancy rate $(75.0 \%)$ (table 1$)$ were both higher than that of $74.2 \%$ and $55.4 \%$ [29], respectively. Our vitrification procedures may need to be modified to increase the vitrified oocyte survival rate post warming and also keep high fertilization and pregnancy rates per transfer in future study. Jones et al also shows that fertilization, blastocyst development, pregnancy and live birth rates are comparable in fresh and vitrified sibling oocytes from the same stimulation cycle [22]. They achieved a good result of $55 \%$ live birth rate in both vitrified oocyte and fresh oocytes groups [22]. In our study, eight healthy babies $(66.7 \%$ of live birth rate) derived from vitrified oocytes in 12 frozen-thawed donor cycles were compared eight healthy babies (57.1\% of live birth rate) from 14 fresh donor cycles in table 1. In conclusion, the simplified oocyte/embryo vitrification and warming protocol (Irvine
Scientific) combined with a vitristraw (SciTech Invention) is a highly efficient vitrification procedure that can be used to freeze human oocytes for future use.

Some studies demonstrate embryos from vitrified oocytes could be re-vitrified and resulted in healthy babies $[16,36$, $37,44]$. In Dorfmann's study, $57 \%$ of clinical pregnancy rate from transfer re-vitrified embryos derived from vitrified donor oocytes was compared $51 \%$ of that from transferring the embryos derived from vitrified donor oocytes [44]. Also, Lamb et al demonstrate there is no statistically significant difference in implantation ( $48 \%$ vs $64 \%$ ), pregnancy ( $66 \%$ vs $70 \%)$ or live birth $(56 \%$ vs $65 \%)$ rates between transferring fresh blastsocysts derived from vitrified oocytes and transferring re-vitrified blastsocysts derived from vitrified oocytes, respectively [36]. Moreover, after studying a large series of transfers of vitrified embryos generated from previously vitrified oocytes, Cobo et al show that double vitrification has no impact on delivery rate [37].

Compared with the $0.43 \%$ of monozygotic twins rate in the general population, the monozygotic twins rate in the IVF increased to $1.02 \%(94 / 9214)$ [45], 2.69\%(93/3,463) [46], or $1.17 \%(122 / 10,470)$ [47], or $2.1 \%(131 / 6223)$ [51]. But the reasons for this are still unclear [53]. By analyzing 93 monozygotic twins from 3,463 pregnant women in IVF cycles, some procedures in assisted reproductive technique, such as the embryo number of transfer, assisted hatching, ICSI and vitrification did not increase probability of monozygotic twins, but extended embryo culture (from day 3 to day 4 and day 5/6) increased the rate of monozygotic twins $(1.27 \%, 3.40 \%, 4.63 \%$ on day 3 , day 4 , day $5 / 6$ transfer, respectively) [46]. Other studies also show the similar result of the increasing monozygotic twinning rate by comparing day 3 and day 5 embryo transfer [47-52]. But, after analyzing 1,951 fresh IVF/ICSI cycles, Papanikolaou, et al found out that the monozygotic twins rate was $2.6 \%(8 / 308)$ in day 3 transfer comparing $1.8 \%(5 / 271)$ in day 5 transfer [54]. Vitthala, et al demonstrate ICSI has 2.25 times higher risk of monozygotic twins than natural conception [50] and Mukaida et al show monozygotic twins rates were $2.7 \%(13 / 486)$ in fresh blastocyst transfer and $4.1 \%(15 / 363)$ in vitrified blastocyst transfer, although there was no statistical difference between two groups [55]. The risk factors of monozygotic twins involved in IVF still need more investigation.

We report the live birth of healthy monozygotic twins (born on January 13, 2015) resulted from a re-vitrified day 5 embryo derived from a vitrified oocyte, which was thawed, fertilized and cultured to a blastocyst. Dose re-vitrified oocytes/blastocyst increase risk of monozygotic twins? That remains a question for further study.

\section{Conclusion}

Vitrification techniques can be used to freeze human oocytes for future use. The fertilization, blastocyst development, clinical pregnancy and live birth rates in frozen/thawed oocyte cycles exhibited little functional difference with those in fresh oocyte cycles. Additionally, 
extra embryos derived from vitrified oocytes can be revitrified. We report the first live birth of healthy monozygotic twins (born on January 13, 2015) resulted from a re-vitrified blastocyst derived from a vitrified oocytes. This result shows that an embryo, after being twice frozen/thawed, can still be split naturally into monozygotic embryos like a fresh embryo after transfer.

\section{Acknowledgements}

The authors acknowledge Ms. Folita Sherman, Ms. Carla Yelton and other staff in NCCRM for their assistance with IVF procedures, and Dr. Glen Hofmann for reviewing this paper.

\section{References}

[1] Chen, C. (1986). Pregnancy after human oocytes cryopreservation. Lancet, 327 (8486), 884-886.

[2] Kuleshva L., et al. (1999). Birth following vitrification of a small number of human oocytes. Human and Reproduction, 14 (12), 3077-3079.

[3] Cao, Y., et al. (2009). Comparison of survival and embryonic development in human oocytes cryopreserved by slowfreezing and vitrification. Fertility and Sterility, 92 (4), 13061311.

[4] Edgar, D., et al. (2012). A critical appraisal of cryopreservation (slow cooling verus vitrification) of human oocytes and embryos. Human Reproduction Update, 18 (5), 536-554.

[5] Smith, G., et al. (2010). Prospective randomized comparison of human oocyte cryopreservation with slow-rate freezing or vitrification. Fertility and Sterility, 94 (6), 2088-2095.

[6] Katayama K., et al. (2003). High survival rate of vitrified human oocytes results in clinical pregnancy. Fertility and Sterility, 80 (1), 223-224.

[7] Kyono, K., et al. (2005). Successful Pregnancy and Delivery After the Transfer of a Single Blastocyst Derived From a Vitrified Mature Human Oocyte. Fertility and Sterility, 84, 1017.e5-e6.

[8] Lucena E., et al. (2013). Successful ongoing pregnancies after vitrification of oocytes. Fertility and Sterility, 86 (1), 108-111.

[9] Garcia-Velasco J., et al. (2013). Five years' experience using oocyte vitirfication to preserve fertility for medical and nonmedical indications. Fertility and Sterility, 99 (7), 19941999.

[10] Braga, D., et al. (2016). Freeze-all, oocyte vitrification, or fresh embryo transfer? Lessons from an egg-sharing donation program. Fertility Sterility, 106 (3), 615-622.

[11] Munck, N., et al. (2016). Open versus closed oocyte vitrification in an oocyte donation programme: a prospective randomized sibling oocyte study. Human Reproduction, 31 (2), 377-383.

[12] Crawford, S., et al. (2017). Cryopreserved oocyte versus fresh oocyte assisted reproductive technology cycles, United States,

\section{Fertility and Sterility, 107 (1), 110-118.}

[13] Choi D. H., et al. (2000). Pregnancy and delivery of healthy infants developed from blastocyts in an IVF-ET program. Fertility and Sterility, 74 (1), 838-839.

[14] Desai N., et al. (2007). Artificial collapse of mouse and human blastocysts prior to vitrification. Fertility and Sterility, 88 (51), 5343-5344.

[15] Hong S., et al. (2009). Cryopreserved human blastsocysts after vitrification result in excellent implantation and clinical pregnancy rates. Fertility and Sterility, 92 (6), 2062-2064.

[16] Cobo A., et al. (2009). Cumullative outcome after transferring "re-vitrified" embryos in ovum donation cycles conducted with vitrified donors oocytes. Fertility and Sterility, 92 (3), 587.

[17] Anderson, R., et al. (2017). Blastocyst vitrification and preimplantation genetic screening (PGS) has facilitated the routine implementation of "cryo-all" cycles: a change in practice management benefiting both the patients and laboratory. Fertility and Sterility, 108 (3), e352-e353.

[18] Forman, E. J., et al. (2102). Oocyte vitrification does not increase the risk of embryonic aneuploidy or diminish the implantation potential of blastsocysts created after intracytoplasmic sperm injection: a novel, paired randomized controlled trial using DNA fingerprinting. Fertility and Sterility, 98 (3), 644-649.

[19] Cobo, Ana. (2012). Oocyte vitrification: a watershed in ART. Fertility and Sterility, 98 (3), 600-601.

[20] Cobo, a., et al. (2008). Comparison of concomitant outcome achieved with fresh and cryopreserved donor oocytes vitrified by the Cryotop method. Fertility and Sterility, 89 (60), 16571664.

[21] Giordana, S. M., et al. (2010). Impact of embryo quality in fresh versus vitrified oocytes in an egg donation program. Fertility and Sterility, 94 (4), s111.

[22] Jones, A. E., et al. (2013). Fresh versus vitrified: results of an egg donor program comparing sibling oocyte outcomes. Fertility and Sterility, 100 (30), s95.

[23] Domingues, T. S., et al. (2014). Egg donation produces similar pregnancy rates with the blastocyst transfer from fresh or vitrified oocytes. Fertility and Sterility, 102 (3), e60.

[24] Homburg, R., et al. (2009). Oocyte vitrification-Women's emancipation set in stone. Fertility and Sterility, 91 (4), 13191320.

[25] Cobo, A., et al. (2013). Is vitrification of oocytes useful for fertility preservation for age-related fertility decline and in cancer patients? Fertility and Sterility, 99 (6), 1485-1495.

[26] Gidoni, Y., et al. (2008). Cryopreservation of a mother's oocytes for possible future use by her daughter with Turner syndrome: case report. Fertility and Sterility, 90 (5), e9-e12.

[27] Rybak, e., et al. (2009). Egg freezing, procreative liberty, and ICSI: the double standards confronting elective self-donation of oocytes. Fertility and Sterility, 92 (5), 1509-1512.

[28] Ethics Committee of the American Society for Reproductive Medicine. (2018). Planned oocyte cryopreservation for women seeking to preserve future reproductive potential: an Ethics Committee opinion. Fertility and Sterility, 110 (6), 1022-1028. 
[29] Cobo A., et al. (2010). Use of cryo-banked oocytes in an ovum donation program: a prospective, randomized, controlled, clinic trial. Human Reproduction, 25, 2239-2246.

[30] Cobo A., et al. (2010). Development of a predictive model for donor's vitrified oocyte 's survival in an egg-bank. Fertility and Sterility, 94 (4), s20

[31] Shu, y., et al. (2013). Single blastocyst transfer in patients utilizing vitrified donor oocytes from a donor bank. Fertility and Sterility, 100 (3), s166.

[32] Quaas, A., et al. (2012). Egg banking in the USA: current status of commercially available cryopreserved oocytes. Fertility and Sterility, 98 (3), s65.

[33] Fung, M. Y., et al. (2015). The advent of frozen egg banks: a comparison of outcomes to fresh donor eggs. Fertility and Sterility, 104 (3), e32.

[34] Hammond, K. R., et al. (2018). Impact of vitrified egg banking on donor oocyte source: is recruitment by practices a dying ART? Fertility and Sterility, 110 (4), e155-e156.

[35] Asimakopoulos, B., et al. (2011). In vitro maturation and fertilization of vitrified immature human oocytes, subsequent vitrification of produced embryos, and embryo transfer after thawing. ? Fertility and Sterility 95 (6), 2123. e1-e2.

[36] Lamb, J., et al. (2018). Outcomes of vitrified donor oocytes: implantation rates are increased when blastocysts are vitrified prior to embryo transfer. Fertility and Sterility, 109 (3), e33e34.

[37] Cobo, A., et al. (2013). Outcome of cryotransfer of embryos developed from vitrified oocytes: double vitrification has no impact on delivery rates. Fertility and Sterility, 99 (6), 16231630 .

[38] Mukaida, T., et al. (2005). Birth After Vitrified Human Oocytes Using Cryoloop Technique. 84 (1), s454

[39] Bonetti, A., et al. (2011). Ultrastructural evaluation of human metaphase II oocytes after vitrification: closed verus open devices. Fertility and Sterility, 95 (3) 928-935.

[40] Taylor, T. H., et al. (2010). Oocyte vitrification: similar results using a straw or cryolock. Fertility and Sterility, 94 (4), s109.

[41] Feng, H. L. and Tsai, T. (2016). Impact of different vitrification devices on oocyte DNA integrity in oocytes. Fertility and Sterility, 106 (3), e140.

[42] Weatherby, F., et al. (2013). Oocyte bank set up by comparing different vitrification devices. Fertility and Sterility, 100 (3), s174.

[43] Mizuno, S., et al. (2014). Comparison of vitrification devices for human embryos between open and closed system. Fertility and Sterility, 102 (3) e122-e123.

[44] Dorfmann, A. D., et al, (2014). Frozen embryo transfers using embryos derived from vitrified donor oocytes from the Fairfax eggbank. 102 (3), e124.

[45] Vaughan, D. A., eat al. (2015). Monozygotic Twinning in IVF: Do They Cluster? Fertility and Sterility, 103 (2) e38-e39.

[46] Liu, H., et al. (2018). Elevated incidence of monozygotic twinning is associated with extended embryo culture, but not with zona pellucida manipulation or freeze-thaw procedure. Fertility and Sterility, 109 (6), 1044-1050.

[47] Tocino, A., et al. (2015). Monozygotic twinning after assisted reproductive technologies: a case report of asymmetric development and incidence during 19 years in an international group of in vitro fertilization clinics. Fertility and Sterility, 103 (5), 1185-1189,

[48] Shipley, S. K., et al. (2003). Factors involved in monozygotic twinning: a three year prospective. Fertility and Sterility, 80 (3), 290-291.

[49] Spandorfer, S. D., et al. (2003). Monozygotic twinning is not increased after single blastocyst transfer compared with single cleavage-stage embryo transfer. Fertility and Sterility, 80 (3), 75-76.

[50] Vitthala S, et al. (2009). The risk of monozygotic twins after assisted reproductive technology: a systematic review and metaanalysis. Human and Reproduction Update, 15 (1), 45-55.

[51] Knopman JM, et al. (2014). What makes them split? Identifying risk factors that lead to monozygotic twins after in vitro fertilization. Fertility and Sterility, 102 (1), 82-89.

[52] Shipley, S. K., et al. (2008). Analysis of biochemical and biophysical risk factors for monozygotic twinning observed within an in vitro fertilization program. Fertility and Sterility, 90, Supplement, s215-s216.

[53] Mateiael, I., et al. (2016). Do ARTs affect the incidence of monozygotic twinning? Human and Reproduction, 15 (11), $2435-2441$

[54] Papanikolaou, E. G., et al. (2010). Monozygotic twinning is not increased after single blastocyst transfer compared with single cleavage-stage embryo transfer. Fertility and Sterility, 93 (2), 592-597.

[55] Mukaida, T., et al. (2007) Does vitrified blastocyst (BL) transfer increase the incidence of monozygotic twinning (MZT)? (analysis of 1,113 cycles for 3 years). Fertility and Sterility, 88 (1), s434. 